\title{
Search for heavy resonances in the dilepton final state with the CMS detector
}

\section{Wenxing Fang* (on behalf of the CMS collaboration)}

School of Physics and Nuclear Energy Engineering, Beihang University, Beijing, China

E-mail: wenxing.fang@cern.ch

\begin{abstract}
A search for a new narrow resonance decaying to an electron pair or a muon pair is performed using $13 \mathrm{TeV}$ proton-proton collision data collected in 2015 by the CMS experiment at the LHC Run 2. The dielectron event sample corresponds to an integrated luminosity of $2.6 \mathrm{fb}^{-1}$ while the dimuon event sample corresponds to an integrated luminosity of $2.8 \mathrm{fb}^{-1}$. The results are interpreted in terms of the possible presence of a new massive narrow spin 1 boson decaying into electron or muon pairs, for different new physics scenarios beyond the Standard Model.
\end{abstract}

Fourth Annual Large Hadron Collider Physics

13-18 June 2016

Lund, Sweden

${ }^{*}$ Speaker. 


\section{Introduction}

The standard model (SM) of particle physics is a tremendously successful theory with a wealth of experimental results supporting it. However, it has some notable omissions such as the lack of a dark matter candidate and the absence of the description of the gravitation force as well as several shortcomings such as the hierarchy problem. Therefore many theories of physics beyond the SM have be proposed to address these issues. Some of these new physics models (e.g. Grand Unification Theories [1], extra spatial dimensions [2]) involve new neutral bosons with masses in the $\mathrm{TeV}$ range, which could decay into a dilepton pair. If such heavy bosons exist they would manifest themselves by the presence a narrow peak in the dilepton invariant mass spectrum that is dominated by the Drell-Yan (DY) process $(q \bar{q} \rightarrow l \bar{l})$ shown in figure 1 .

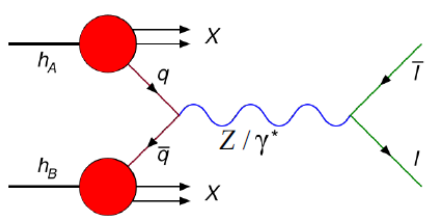

Figure 1: Drell-Yan process diagram.

\section{Event selection}

Using the full dataset collected by the CMS experiment [3] in 2015, the events are first selected by online level 1 and hight level triggers. Then, events with two high energy electrons (muons) are selected offline, each of them with $E_{T}>35 \mathrm{GeV}\left(E_{T}>53 \mathrm{GeV}\right)$, where $E_{T}$ is defined as $E_{T}=E \cdot \sin (\theta)$. Finally identification and isolation criteria are applied to the high energy electrons (muons) to suppress backgrounds. For the dielectron channel, the events are split into 2 categories : the barrel-barrel and the barrel-endcap category.

\section{Standard model Background}

The DY process is the main (irreducible) background to the signal. However other processes (reducible backgrounds) have non negligible contribution: $W+$ jets and multijet events from QCD processes (where jets are misidentified as prompt leptons) and top quark pair production events $(t \bar{t})$, where the top quarks decay leptonically, as well as a contribution from diboson production $(W W, W Z, Z Z)$. Data-driven methods have been designed to estimate the contamination of the multijet background using the "fake rate" method. The $t \bar{t}$ background is estimated from simulation and is cross-checked using the " $e \mu$ " method. The details of the selection criteria and the background estimation are given in reference [4].

\section{Results}

The observed mass spectra together with the predicted SM backgrounds are shown in figure 2 for both the dielectron (barrel-barrel and barrel-endcap categories combined) and the dimuon chan- 
nels. Figure 3 shows the dielectron mass spectra for the barrel-barrel and barrel-endcap categories separately. The observed mass spectra agree well with these of the predicted SM backgrounds.
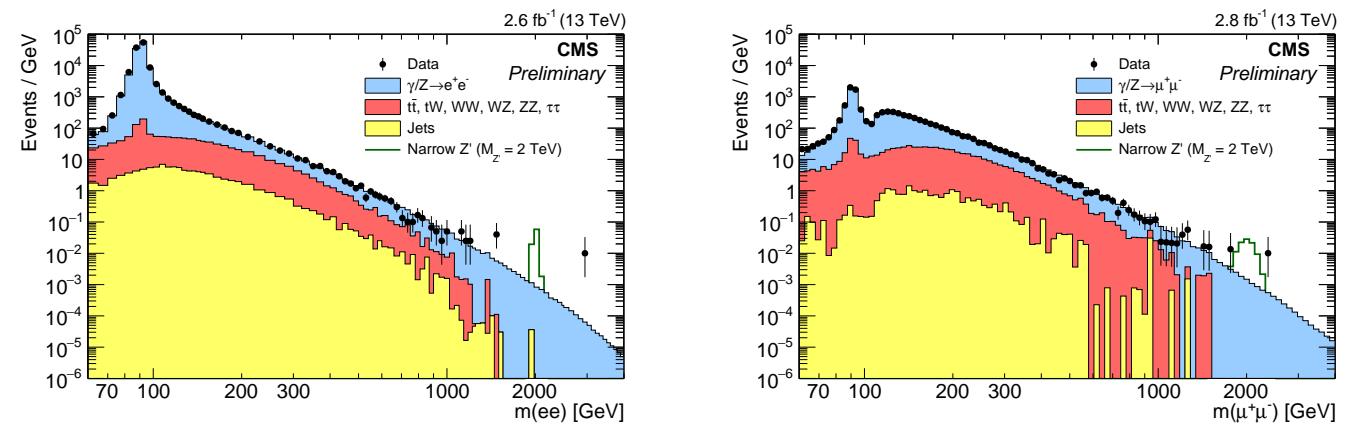

Figure 2: Invariant mass spectrum of $e e$ (left) and $\mu^{+} \mu^{-}$(right) events. The points with error bars represent the data. The histograms represent the expectations from standard model processes: $Z / \gamma^{*}, t \bar{t}$ and other sources of prompt leptons as well as the jets background which contains at least one jet that has been misreconstructed as a lepton.
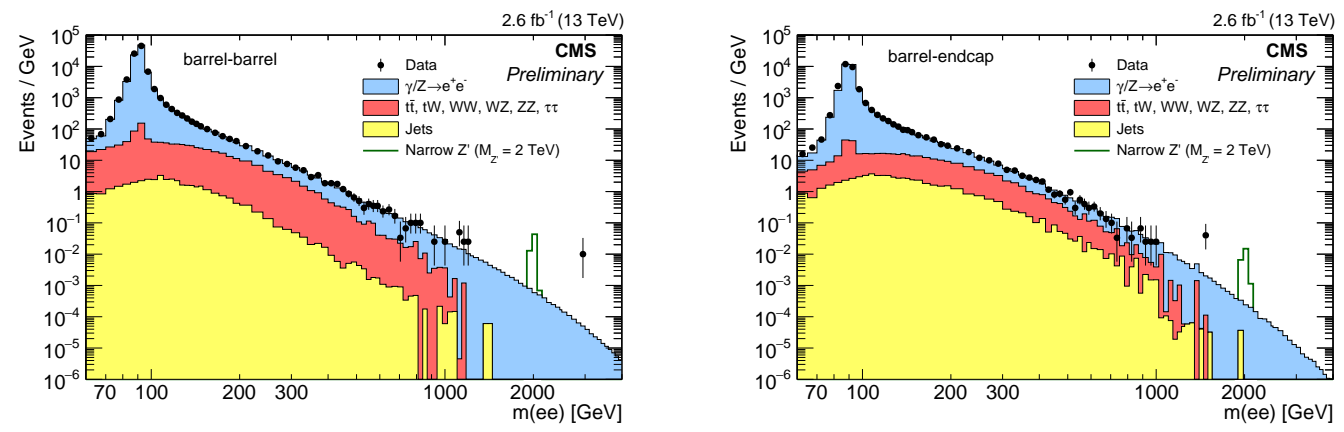

Figure 3: Invariant mass spectrum of $e e$ events split into barrel-barrel (left) and barrel-endcap (right) categories. The points with error bars represent the data. The histograms represent the expectations from standard model processes: $Z / \gamma^{*}, t \bar{t}$ and other sources of prompt leptons as well as the jets background which contains at least one jet that has been misreconstructed as a lepton.

Since no new physics is observed, limits are set on possible contributions from narrow heavy resonances. The variable under study is the ratio of the production cross section times branching ratio to two leptons for new heavy boson of spin 1 and the $\mathrm{Z}$ boson. The advantage of taking a ratio is that several sources of systematic uncertainty cancel, including the one related to the integrated luminosity. The results are shown in figure 4 (for the $e e$ and $\mu^{+} \mu^{-}$channels separately) as well as figure 5 (for the $e e$ and $\mu^{+} \mu^{-}$channels combined).

The lower limits on the resonance mass are given in table 1 . A sequential standard model $Z_{S S M}^{\prime}$ and a superstring-inspired $Z_{\psi}^{\prime}$ lighter than $3.15 \mathrm{TeV}$ and $2.6 \mathrm{TeV}$ respectively are excluded at $95 \%$ confidence level. 

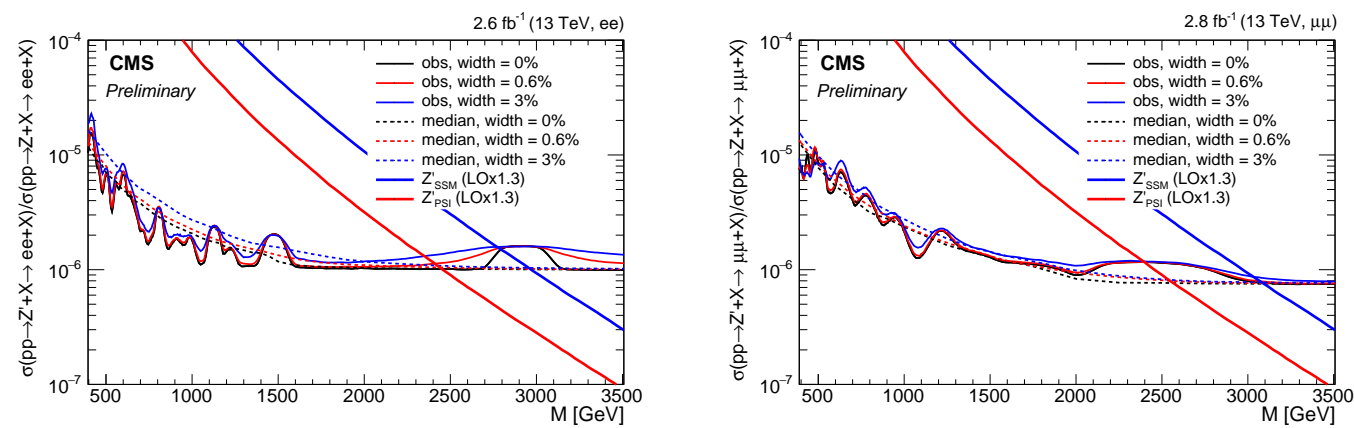

Figure 4: The observed limits obtained at $95 \%$ confidence level on the cross section ratio $\left(Z^{\prime} / Z\right)$ for various $Z^{\prime}$ widths for the dielectron channel (left) and dimuon channel (right).
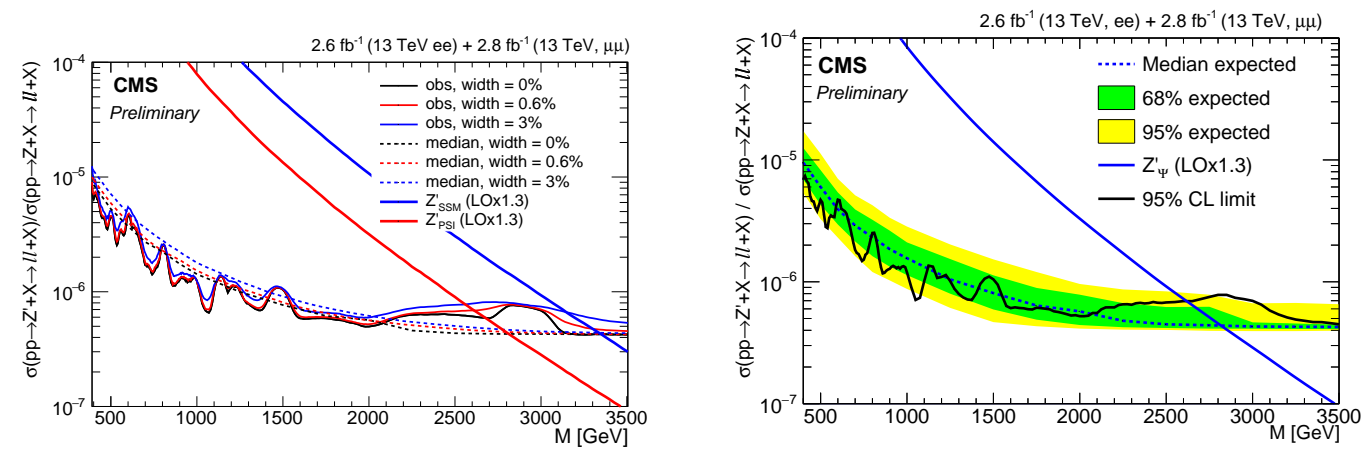

Figure 5: The observed limits obtained at $95 \%$ confidence level on the cross section ratio $\left(Z^{\prime} / Z\right)$ for various $Z^{\prime}$ widths for the dielectron and dimuon channels combined (left) and with the expected limits bands included (right).

\begin{tabular}{|c|c|c|c|c|}
\hline \multirow{2}{*}{ channel } & \multicolumn{2}{|c|}{$Z_{\psi}^{\prime}$} & \multicolumn{2}{c|}{$Z_{S S M}^{\prime}$} \\
\cline { 2 - 5 } & observed & expected & observed & expected \\
\hline$e e$ & 2.40 & 2.45 & 2.75 & 2.95 \\
\hline$\mu^{+} \mu^{-}$ & 2.40 & 2.55 & 3.00 & 3.05 \\
\hline$e e+\mu^{+} \mu^{-}$ & 2.60 & 2.80 & 3.15 & 3.35 \\
\hline
\end{tabular}

Table 1: The observed and expected mass limits(in TeV) on the $Z_{S S M}^{\prime}$ and $Z_{\psi}^{\prime}$ bosons.

\section{References}

[1] A. Leike, The Phenomenology of Extra Neutral Gauge Bosons, Phys. Rept. 317 (1999) 143, [hep-ph/9805494]

[2] L. Randall, R. Sundrum, A Large Mass Hierarchy from a Small Extra Dimension, Phys. Rev. Lett. 83 (1999) 3370, [hep-ph/9905221]

[3] The CMS Collaboration, The CMS experiment at the CERN LHC, JINST 3:S08004, 2008.

[4] The CMS Collaboration, Search for a narrow resonance produced in $13 \mathrm{TeV}$ pp collisions decaying to electron pair or muon pair final states, CMS PAS EXO-15-005 